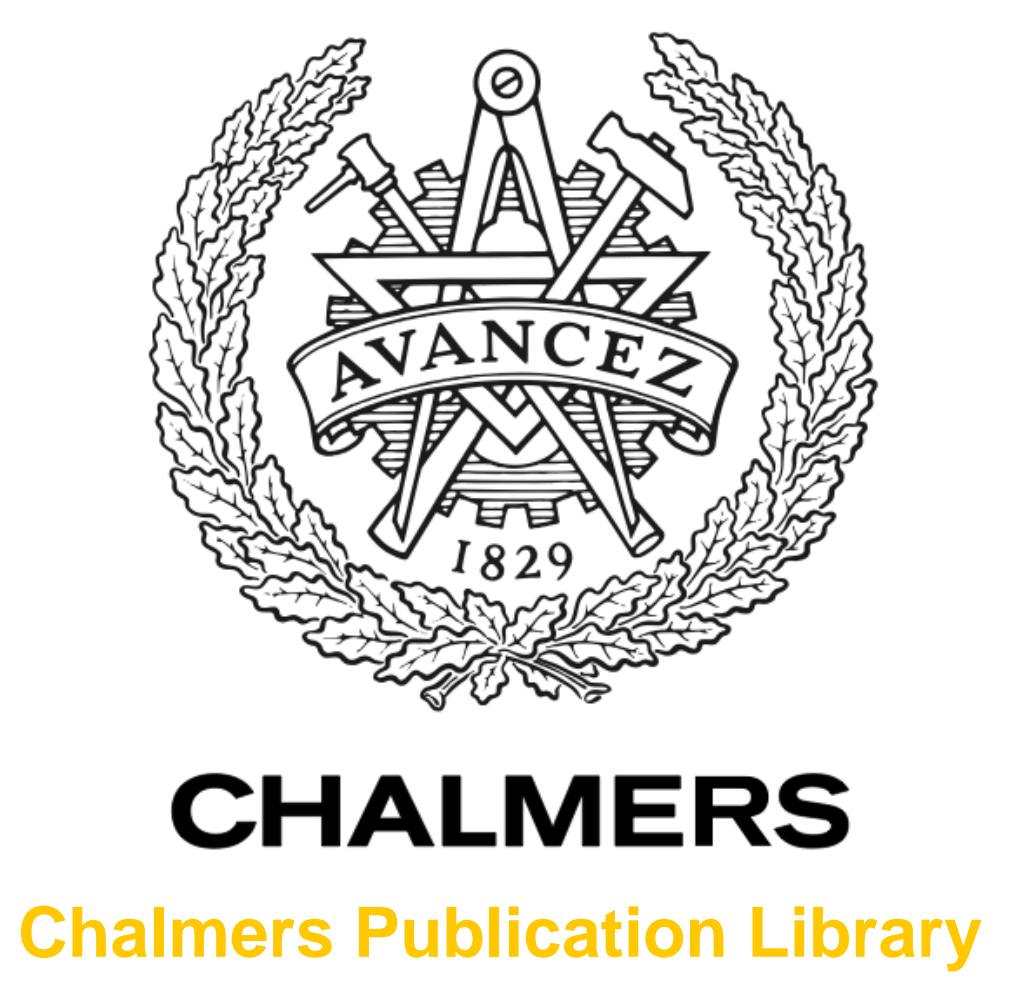

\title{
Electric field effects on spin accumulation in Nb-doped SrTiO3 using tunable spin injection contacts at room temperature
}

This document has been downloaded from Chalmers Publication Library (CPL). It is the author's version of a work that was accepted for publication in:

Applied Physics Letters (ISSN: 0003-6951)

Citation for the published paper:

Dash, S. ; Kamerbeek, A. ; de Vries, E. (2014) "Electric field effects on spin accumulation in $\mathrm{Nb}$-doped SrTiO3 using tunable spin injection contacts at room temperature". Applied

Physics Letters, vol. 104(21),

http://dx.doi.org/10.1063/1.4880895

Downloaded from: http://publications.lib.chalmers.se/publication/198716

Notice: Changes introduced as a result of publishing processes such as copy-editing and formatting may not be reflected in this document. For a definitive version of this work, please refer to the published source. Please note that access to the published version might require a subscription. 


\title{
Electric field effects on spin accumulation in $\mathrm{Nb}$-doped $\mathrm{SrTiO}_{3}$ using tunable spin injection contacts at room temperature
}

\author{
A. M. Kamerbeek, ${ }^{1}$ E. K. de Vries, ${ }^{1}$ A. Dankert, ${ }^{2}$ S. P. Dash, ${ }^{2}$ B. J. van Wees, ${ }^{1}$ \\ and T. Banerjee ${ }^{1, a)}$ \\ ${ }^{1}$ Physics of Nanodevices, Zernike Institute for Advanced Materials, University of Groningen, \\ Nijenborgh 4, 9747 AG Groningen, The Netherlands \\ ${ }^{2}$ Department of Microtechnology and Nanoscience, Chalmers University of Technology, \\ SE-41296 Gothenburg, Sweden
}

(Received 30 April 2014; accepted 19 May 2014; published online 30 May 2014)

\begin{abstract}
We report on features in charge transport and spin injection in an oxide semiconductor, $\mathrm{Nb}$-doped $\mathrm{SrTiO}_{3}$. This is demonstrated using electrically tunable spin injection contacts which exploit the large electric field at the interface and its interplay with the relative permittivity of the semiconductor. We realize spin accumulation in $\mathrm{Nb}$-doped $\mathrm{SrTiO}_{3}$ which displays a unique dependence of the spin lifetime with bias polarity. These findings suggest a strong influence of the interface electric field on the charge transport as well as on spin accumulation unlike in conventional semiconductors and opens up promising avenues in oxide spintronics. (C) 2014 AIP Publishing LLC. [http://dx.doi.org/10.1063/1.4880895]
\end{abstract}

The interplay between the electron, spin, lattice, and orbital degrees of freedom at complex oxide heterointerfaces have led to a rich variety of physical phenomena, often nonexistent in their bulk. ${ }^{1}$ At heterointerfaces of two insulating oxide thin films that involves the dielectric oxide $\mathrm{SrTiO}_{3}$, a tunable two-dimensional conducting channel which is either magnetic or superconducting has been demonstrated. ${ }^{2-4}$ Such interfaces, crucial for the development of oxide electronics, have been widely studied in the last decade. On the other hand, interfaces with oxide semiconductors where both the electron spin as well as its charge can be exploited in new devices is less studied but particularly useful for the nascent field of oxide spintronics. Semiconducting $\mathrm{SrTiO}_{3}$ can be realized by doping $\mathrm{Nb}$ at the Ti site. This n-type semiconductor is characterized by unconventional electrical conductivity, ${ }^{5,6}$ compared to its counterparts such as $\mathrm{Si}, \mathrm{Ge}$, and GaAs and arises due to the unusual response of its relative permittivity with temperature and electric field.

It is in this pursuit that we design tunable spin injection contacts and investigate spin injection in $\mathrm{Nb}$-doped $\mathrm{SrTiO}_{3}$ $\left(\mathrm{Nb}: \mathrm{SrTiO}_{3}\right)$ at room temperature. For this, we use the three terminal (3T) geometry which has been successfully used to inject and detect spin accumulation in conventional semiconductors such as GaAs, Si, and Ge. ${ }^{7-9}$ An important prerequisite to realize efficient spin injection into a semiconductor is direct tunneling transport of the spin polarized carriers from the ferromagnet (FM) to the semiconductor (S), which is often impeded by the formation of Schottky barriers at the $\mathrm{FM} / \mathrm{S}$ interfaces.

Recently, spin injection in a two dimensional electron gas between $\mathrm{LaAlO}_{3}$ and $\mathrm{SrTiO}_{3}$ and in a highly doped $\mathrm{SrTiO}_{3}$ semiconductor using a $3 \mathrm{~T}$ geometry have been reported. ${ }^{10,11}$ The latter study uses a relatively thick tunnel barrier that fully dominates the device resistance and washes off intricate correlation effects usually prevalent at interfaces with oxide semiconductors. In this work, we report on a

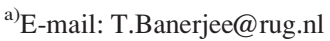

different route to efficiently inject spins in oxide semiconductors which is related to the electric field dependence of the relative permittivity of $\mathrm{Nb}: \mathrm{SrTiO}_{3}$ at the interface with tunable spin injection contacts. The tunable spin contacts comprise of a ferromagnet as a source of spin polarized carriers and a thin tunnel barrier of varying thickness. A tunnel barrier of appropriate thickness tunes the charge transport mechanism to be tunneling dominated which leads to the observation of spin injection in $\mathrm{Nb}: \mathrm{SrTiO}_{3}$, while retaining a large electric field at the semiconductor surface. Using such tunable contacts, we find features in the electrical and spin injection characteristics of the devices that are not observed with conventional semiconductors and opens up promising routes for spintronics using complex oxides.

For realizing efficient spin injection into $\mathrm{Nb}: \mathrm{SrTiO}_{3}$, we fabricated $\mathrm{Co} / \mathrm{AlO}_{\mathrm{x}}(t) / \mathrm{Nb}: \mathrm{SrTiO}_{3}$ contacts (Fig. 1(a)) where the thickness $t$ of the Al layer was varied $(0,7 \pm 1$, $11 \pm 1 \AA$ ). This tunes the charge transport and enables tunneling by reducing the Schottky barrier height and width. ${ }^{12}$ $\mathrm{Nb}: \mathrm{SrTiO}_{3}(0.1 \mathrm{wt}$. \% Nb) substrates were treated using a standard chemical etching protocol to achieve a $\mathrm{TiO}_{2}$ terminated surface. ${ }^{13}$ A thin Al layer was deposited at a pressure $\sim 1 \times 10^{-6}$ Torr using an electron-beam evaporator followed by in situ oxidation in oxygen plasma. Subsequently, a 20 nm thick cobalt and gold layer were grown. Post processing with standard ultraviolet lithography and ion-beam etching was used to pattern the spin injection pillars with sizes ranging from 50 to $200 \times 200 \mu \mathrm{m}^{2}$. Atomic force microscopy on the surface of a spin injection contact shows a root-mean-square surface roughness of $\sim 4 \AA$, whereas for the treated substrate it is $\sim 1.8 \AA$.

To study the electrical characteristics of the spin contacts, Current-Voltage $(I-V)$ measurements in a 3T geometry (Fig. 1(a)) were performed. In Fig. 1(b), the room temperature $I-V$ characteristics of the three different spin contacts are shown. This shows a large tunability of the interface resistance as well as a gradual reversal of the $I-V$ asymmetry with increasing tunnel barrier thickness. Such changes in $I-V$ 
(a)

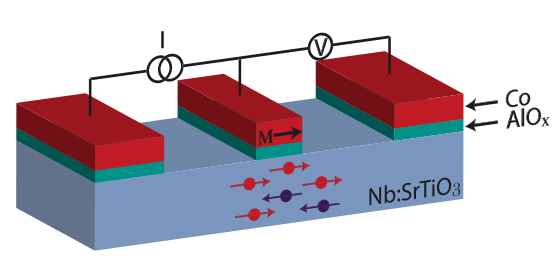

(c)

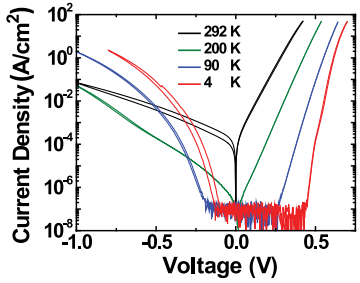

(d)

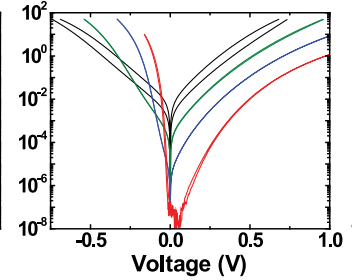

(b)

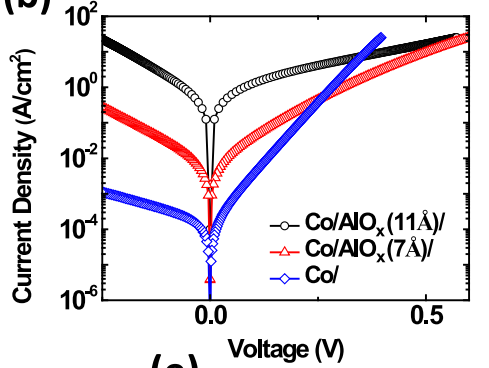

(e)

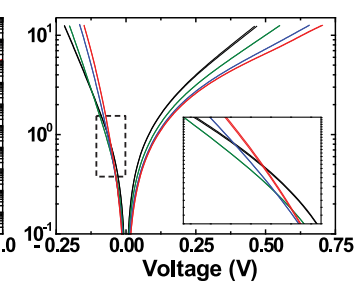

FIG. 1. (a) Schematic of $3 T$ geometry for $I-V$ and Hanle measurements. (b) $I-V$ characteristics of the three spin contacts at room temperature. Temperature dependent $I-V$ measurement of (c) $\mathrm{Co} / \mathrm{Nb}: \mathrm{SrTiO}_{3}$, (d) $\mathrm{Co} / \mathrm{AlOx}$ $(7 \AA) / \mathrm{Nb}: \mathrm{SrTiO}_{3}$, and (e) $\mathrm{Co} / \mathrm{AlOx}(11 \AA) / \mathrm{Nb}: \mathrm{SrTiO}_{3}$ interface. The inset shows the $I-V$ in the dashed box. The crossing shows the increase of current at reverse bias at lower temperatures. Same colors denote the same measurement temperatures in (c)-(e). characteristics resemble earlier works of engineered spin contacts on $\mathrm{Si}$, where the Schottky barrier height is reduced by low work function materials at the semiconductor interface. ${ }^{14}$ This is in contrast to our work where we realize such tunability by insertion of a thin tunnel barrier of varying thickness. To gain a better understanding of this tunability, we performed temperature dependent $I-V$ measurements (4-292 K) shown in Figs. 1(c)-1(e). At room temperature, the $\mathrm{Co} / \mathrm{Nb}: \mathrm{SrTiO}_{3}$ interface exhibits rectification (Fig. 1(b)) as well as a significant reduction of the forward current when decreasing temperature. Using the standard thermionic emission (TE) model, we extract the Schottky barrier height $\phi_{b}=0.63 \pm 0.02 \mathrm{eV}$ and the ideality factor $\mathrm{n}=1.3$ at room temperature. At reverse bias, the current initially decreases upon cooling, however, an anomalous enhancement is observed below $200 \mathrm{~K}$. The $I-V$ measurement displays a hysteresis, predominantly at reverse bias, reminiscent of the colossal electro-resistance effect. ${ }^{15}$ With the introduction of $7 \AA \mathrm{AlO}_{\mathrm{x}}$ layer, an increase in the forward current is observed with no rectification at room temperature as shown in Fig. 1(d). The forward current decreases with decreasing temperature but does not show the exponential dependence with increasing forward bias as in Fig. 1(c). At negative bias, a large increase in current is observed with decreasing temperature except at very low bias $(\mathrm{V} \geq-15 \mathrm{mV})$. For the thickest tunnel barrier ( $11 \AA$ ), we observe an increase in the current in both bias directions compared to Fig. 1(d). The increase of current at reverse bias when lowering the temperature is visible from the crossing of the curves around $-80 \mathrm{mV}$ in the inset of Fig. 1(e). This signifies the existence of a Schottky barrier, although reduced in height and width.

To qualitatively analyze the $I-V$ characteristics we use the well-known transport processes for metal/semiconductor interfaces. ${ }^{12}$ Generally, three transport mechanisms are possible depending on the potential landscape of the interface: (1) TE: the excitation of charge carriers over the Schottky barrier by thermal energy, (2) Thermally Assisted Fieldemission (TFE): tunneling of charge carriers through the barrier at energies higher than the Fermi level due to thermal excitation, (3) Field-Emission (FE): (tunneling) where charge carriers tunnel through the potential barrier around the Fermi energy of the system (Fig. 2(a)).
Compared to conventional n-doped degenerate semiconductors the rather large rectification of the $\mathrm{Co} / \mathrm{Nb}: \mathrm{SrTiO}_{3}$ interface is surprising. ${ }^{16,17}$ This originates from the large dielectric permittivity of $\mathrm{Nb}: \mathrm{SrTiO}_{3}(\sim 330$ at room temperature) widening the depletion region. Standard analysis of the temperature dependent $I-V$ measurement shows the charge transport to be dominated by thermally assisted fieldemission. The pronounced increase in reverse current with decreasing temperature signifies an increase in fieldemission, being the only transport mechanism not requiring thermal energy. Such behavior, although not observed in conventional semiconductors, is commonly observed for $\mathrm{Nb}: \mathrm{SrTiO}_{3}$, as in $\mathrm{Au} / \mathrm{Nb}: \mathrm{SrTiO}_{3}$ diodes. ${ }^{18,19}$ This can be explained by the unique dependence of the relative permittivity $\left(\varepsilon_{r}\right)$ of $\mathrm{Nb}: \mathrm{SrTiO}_{3}$ on electric field and temperature. It is well known that $\varepsilon_{r}$ of $\mathrm{SrTiO}_{3}$ is sensitive to electric field, decreasing with increasing field strength. ${ }^{20}$ Large electric fields, up to several $\mathrm{MeV} / \mathrm{cm}$, develop at the interface of $\mathrm{Nb}: \mathrm{SrTiO}_{3}$ due to band bending. ${ }^{18}$ This reduces $\varepsilon_{r}$ at the
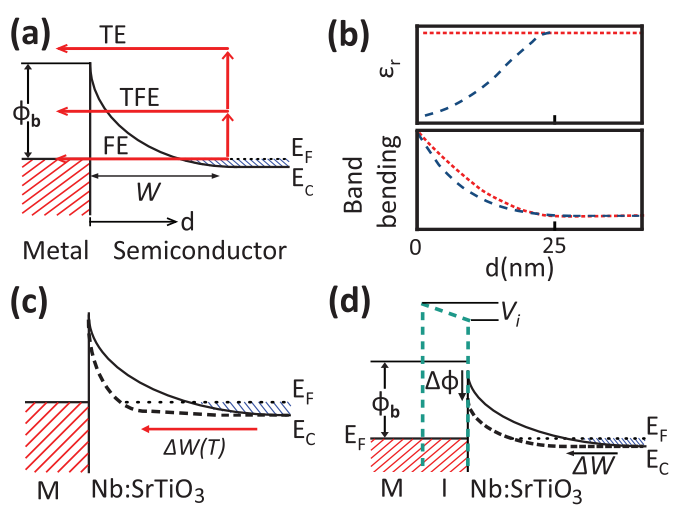

FIG. 2. (a) Energy diagram of metal-semiconductor interface showing the three possible transport mechanisms as discussed in the text. (b) Top panel sketches the relative permittivity $\left(\varepsilon_{r}\right)$ at room temperature with distance from the interface $d$. Bottom panel shows band bending at the semiconductor interface. The short-dashed red line represents a constant permittivity of 330 , the long-dashed blue line shows the modified $\varepsilon_{r}$ due to the built-in electric field and the resulting change in barrier profile. (c) With reducing temperature, $\varepsilon_{r}$ becomes increasingly sensitive to electric field causing a strong narrowing of the depletion width $(\Delta W)$, enhancing tunneling transport. (d) Metal-semiconductor interface (black-solid line) and the effect of inserting a thin tunnel barrier (green/black-dashed line). 
interface as shown in the top panel of Fig. 2(b). The reduction of $\varepsilon_{r}$ will lead to a narrowing of the depletion region $(W)$ according to $W=\left(2 \varepsilon_{r} \phi_{b} / \mathrm{q} N_{d}\right)^{1 / 2}$, where $N_{d}$ is the donor density (bottom panel Fig. 2(b)). This narrowing of the depletion region becomes temperature dependent due to the strong increase of the bulk permittivity, from $\sim 330$ to $>10^{4}$ at $4 \mathrm{~K}$. At the same time, the interface permittivity becomes increasingly sensitive to electric fields, reducing by $3-4$ orders of magnitude compared to the bulk value $\left(>10^{4}\right)$ at low temperatures, which leads to a considerable narrowing of the depletion region (Fig. 2(c)). ${ }^{19}$ Therefore, a large increase in tunneling transport occurs upon cooling if the initial depletion region is thin enough, as is the case for the $0.1 \mathrm{wt} \% \mathrm{Nb}$ doping.

The insertion of a thin $\mathrm{AlO}_{\mathrm{x}}$ tunnel barrier reduces the interface resistance with a gradual reversal of the $I-V$ asymmetry. The origin of this also lies in the strong tunability of the interface permittivity. At the FM/S Schottky interface, all the voltage drops at the semiconductor side. When a thin insulator (I) is introduced between them, this voltage now partially drops over the tunnel barrier $\left(V_{i}\right)$, reducing the voltage drop in the semiconductor and thus the Schottky barrier height $\left(\Delta \phi_{b}\right)$ and width $(\Delta W)$ (Fig. 2(d)). ${ }^{12} \mathrm{In} \mathrm{Nb}: \mathrm{SrTiO}_{3}$, this effect is much larger for two reasons: (i) the relatively high $\varepsilon_{r}$ compared to conventional semiconductors causes a larger portion of the voltage to drop over the tunnel barrier and (ii) due to a reduction of the Schottky barrier the built-in electric field is reduced which increases the interface permittivity of $\mathrm{Nb}: \mathrm{SrTiO}_{3}$ further. These two effects result in a larger voltage drop over the tunnel barrier than with similar contacts on conventional semiconductors and allows for a large tuning of the device resistance. With increasing thickness of the tunnel barrier, the device resistance and behavior becomes less sensitive to changes in the permittivity of $\mathrm{Nb}: \mathrm{SrTiO}_{3}$.

For the contact with a $7 \AA$ barrier, the large increase in reverse current at lower temperature reveals a narrowing of the Schottky barrier width so that transport is tuned from thermally assisted field-emission to field-emission. The strongly reduced temperature sensitivity of the $11 \AA \mathrm{AlO}_{\mathrm{x}}$ interface indicates direct tunneling as the main transport mechanism at all temperatures. With increasing forward bias the current flow should be limited by the added series resistance of the tunnel barrier compared to the Schottky interface. ${ }^{16}$ This trend is indeed observed at high positive bias in Fig. 1(b).
To investigate the suitability of such spin injection contacts at room temperature, we use the 3T geometry (Fig. 1(a)). In such a geometry, the same FM/I contact is used for spin injection and detection. A constant current is sourced through the central (spin injection) contact. This results in spin injection underneath the contact, splitting the electrochemical potential for spin up $\left(\mu_{\uparrow}\right)$ and spin down $\left(\mu_{\downarrow}\right)$, creating a spin accumulation $\Delta \mu=\mu_{\uparrow}-\mu_{\downarrow}$ in the semiconductor (Fig. 3(a)). The spin orientation is controlled by the magnetization direction $(M)$ of the injection contact which, due to shape anisotropy, points in-plane. The application of an outof-plane magnetic field $\left(B_{z}\right)$ will cause Larmor precession of the accumulated spins, de-phasing the spin accumulation (insets of Fig. 3(b)). This is measured by probing the voltage of the central contact relative to the reference contact on the right. The detected voltage thus measures the decay of spin accumulation at the point of injection, in response to an increasing transverse magnetic field and is known as the Hanle effect. The change in voltage $\Delta V$ is related to the spin accumulation by $\Delta V=\gamma \Delta \mu / 2, \gamma$ being the tunnel spin polarization of the $\mathrm{Co} / \mathrm{AlO}_{\mathrm{x}}$ interface. The reduction of the spin accumulation with magnetic field can be approximated by the Lorentzian equation $\Delta \mu(\mathrm{B})=\Delta \mu(0) /\left(1+\left(\omega_{L} \tau\right)^{2}\right)$, where $\omega_{L}$ is the Larmor frequency and $\tau$ the lower bound spin lifetime. $^{9}$

The Hanle effect is observed for the $\mathrm{Co} / \mathrm{AlO}_{\mathrm{x}}(11 \AA) /$ $\mathrm{Nb}: \mathrm{SrTiO}_{3}$ contact. At room temperature and for $+10 \mathrm{~mA}$ bias, a decrease of the spin voltage with a Lorentzian line shape is observed as shown in Fig. 3(b) (black circles). By fitting the data for low field values (red line in Fig. 3(b)), with the Lorentzian equation, a lower bound spin lifetime $\tau=14.1 \pm 0.4 \mathrm{ps}$ and spin voltage $\Delta V \sim 270 \mu \mathrm{V}$ were obtained. Application of an in-plane field shows a small increase in the spin voltage (Fig. 3(b), blue triangles). At negative bias, the Hanle measurement shows a broad magneto-resistance as shown in Fig. 2(c) for a bias current of $-3 \mathrm{~mA}$. Fitting with the Lorentzian equation yields a lifetime of $\sim 3.5$ ps. An exponentially changing background voltage at constant bias current (increasing for $-\mathrm{I}$, decreasing for $+\mathrm{I}$ ) was subtracted from the Hanle measurements. Similar behavior was observed for the different devices that were measured.

The spin voltage is anomalously enhanced compared to that expected from theory by at least 100 times, as is generally observed in 3T Hanle studies. ${ }^{14}$ Although a precise understanding of the enhancement is lacking, the exact
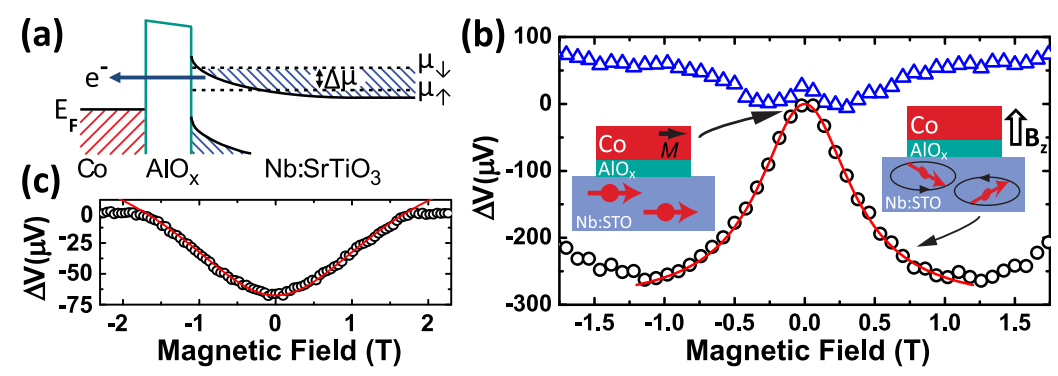

FIG. 3. (a) Energy band diagram of a forward biased metal-insulator-semiconductor interface. Spin accumulation inside $\mathrm{Nb}$ :SrTiO $_{3}$, represented by the splitting of the electrochemical potential $\Delta \mu$ for spin up $\mu_{\uparrow}$ and down $\mu_{\downarrow}$. (b) Out-of-plane Hanle (black circles) and in-plane (blue triangles) measurements at room temperature of the $\mathrm{Co} / \mathrm{AlO}_{\mathrm{x}}(11 \AA) / \mathrm{Nb}: \mathrm{SrTiO}_{3}$ interface at $+10 \mathrm{~mA}$ bias current. The red line is the Lorentzian fit at low fields. A spin voltage $\Delta \mathrm{V} \sim 270 \mu \mathrm{V}$ and a spin lifetime $\tau$ of $14.1 \mathrm{ps}$ are obtained. (c) Hanle measurement at $-3 \mathrm{~mA}$ showing a very broad response and a spin lifetime of $\sim 3.5 \mathrm{ps}$. 
nature of the tunneling process is believed to be a key factor. For instance, such an enhancement is commonly attributed to spin accumulation in interface states or to transport via double step tunneling. ${ }^{8,21}$ However, the former is only possible when these states are decoupled from the semiconductor bulk by a large enough barrier resistance. ${ }^{8,22}$ Further, the obtained spin lifetime is considerably lower than the recently reported value of $\sim 80-100 \mathrm{ps}$ for $0.7 \mathrm{wt}$. $\% \mathrm{Nb}: \mathrm{SrTiO}_{3} .{ }^{11} \mathrm{It}$ might be noted that lifetimes of over $10 \mathrm{~ns}$ at room temperature have recently been calculated for strained $\mathrm{SrTiO}_{3-\delta}{ }^{23}$ Considerably shorter lifetimes than calculated are frequently reported with the $3 \mathrm{~T}$ geometry. ${ }^{14}$ However, the low value of $14.1 \pm 0.4 \mathrm{ps}$, especially compared to the $0.7 \mathrm{wt}$. \% study, is surprising, indicating a different origin of spin de-phasing. Magnetic stray fields due to interface roughness, causing spin de-phasing, can be ruled out since the roughness $\sim 4 \AA$ of the layer stack surface, is lower than or comparable to other works. ${ }^{11,24}$

We believe the origin of the short spin lifetime and its dependence on bias polarity to be related to the electric-field at the interface as well as its coupling with $\varepsilon_{r}$. For instance, it has been shown that the presence of such an electric field results in a cubic Rashba spin-orbit field which can cause additional spin de-phasing. ${ }^{25,26}$ Our findings are different from the recently reported work in Ref. 11, primarily due to the difference of the injection contacts: the $2 \mathrm{~nm}$ thick $\mathrm{MgO}$ tunnel barriers completely dominate the contact resistance and no significant electric field effects are thus expected inside the semiconductor. Further, it has been shown that the spin voltage scales with tunnel barrier resistance and might measure spin signals related to the tunnel contacts instead of the semiconductor. ${ }^{27-29}$ The low spin lifetime, in our work, suggests that the spin accumulation occurs at, or is considerably influenced by, the semiconductor interface properties.

We have carried out Hanle measurements with other contacts such as $\mathrm{Co} / \mathrm{Nb}: \mathrm{SrTiO}_{3}$ and $\mathrm{Co} / \mathrm{AlOx}(7 \AA) / \mathrm{Nb}: \mathrm{SrTiO}_{3}$. Whereas, we find no Hanle effect at bias current up to $+10 \mathrm{~mA}$ in the former, we see the spin voltage is dominated by a large parabolic background in the latter, thus making it difficult to extract a value for the spin lifetime.

We have shown that the electrical characteristics of devices on $0.1 \mathrm{wt}$. \% $\mathrm{Nb}: \mathrm{SrTiO}_{3}$ semiconductor can be significantly tuned by the thickness of the tunnel barrier, unlike in conventional semiconductors. Using such tunable spin injection contacts, we demonstrate unique features in the spin signal and its response to bias polarity. We believe this arises due to the electric field at the interface as well as its coupling with the dielectric permittivity of $\mathrm{Nb}: \mathrm{SrTiO}_{3}$. Our results suggest that the manipulation of charge and spin transport by an electric field at the interface with $\mathrm{Nb}: \mathrm{SrTiO}_{3}$ is a promising route for oxide electronic and spintronic devices.

A.M.K. and T.B. acknowledge financial support from the Netherlands Organization for Scientific Research NWO-VIDI program and the Rosalind Franklin Fellowship and thank K. G. Rana for substrate characterization studies. S.P.D. and A.D. acknowledge financial support from NanoAoA at Chalmers University of Technology.

${ }^{1}$ P. Zubko, S. Gariglio, M. Gabay, P. Ghosez, and J. Triscone, Annu. Rev. Condens. Matter Phys. 2, 141 (2011).

${ }^{2}$ A. Ohtomo and H. Y. Hwang, Nature 427, 423 (2004).

${ }^{3}$ A. Brinkman, M. Huijben, M. van Zalk, J. Huijben, U. Zeitler, J. C. Maan, W. G. van der Wiel, G. Rijnders, D. H. A. Blank, and H. Hilgenkamp, Nature Mater. 6, 493 (2007).

${ }^{4}$ N. Reyren, S. Thiel, A. D. Caviglia, L. Fitting Kourkoutis, G. Hammerl, C. Richter, C. W. Schneider, T. Kopp, A.-S. Rüetschi, D. Jaccard, M. Gabay, D. A. Muller, J.-M. Triscone, and J. Mannhart, Science 317, 1196 (2007).

${ }^{5}$ A. Spinelli, M. A. Torija, C. Liu, C. Jan, and C. Leighton, Phys. Rev. B 81, 155110 (2010).

${ }^{6}$ K. G. Rana, V. Khikhlovskyi, and T. Banerjee, Appl. Phys. Lett. 100, 213502 (2012).

${ }^{7}$ X. Lou, C. Adelmann, M. Furis, S. A. Crooker, C. J. Palmstrøm, and P. A. Crowell, Phys. Rev. Lett. 96, 176603 (2006).

${ }^{8}$ M. Tran, H. Jaffrès, C. Deranlot, J.-M. George, A. Fert, A. Miard, and A. Lemaitre, Phys. Rev. Lett. 102, 036601 (2009).

${ }^{9}$ S. P. Dash, S. Sharma, R. S. Patel, M. P. de Jong, and R. Jansen, Nature 462, 491 (2009).

${ }^{10}$ N. Reyren, M. Bibes, E. Lesne, J.-M. George, C. Deranlot, S. Collin, A. Barthélémy, and H. Jaffrès, Phys. Rev. Lett. 108, 186802 (2012).

${ }^{11}$ W. Han, X. Jiang, A. Kajdos, S.-H. Yang, S. Stemmer, and S. S. P. Parkin, Nature Comm. 4, 2134 (2013).

${ }^{12}$ S. M. Sze and K. K. Ng, Physics of Semiconductor Devices (Wiley, Hoboken, NJ, USA 2006).

${ }^{13}$ G. Koster, B. L. Kropman, G. J. H. M. Rijnders, D. H. A. Blank, and H. Rogalla, Appl. Phys. Lett. 73, 2920 (1998).

${ }^{14}$ R. Jansen, S. P. Dash, S. Sharma, and B. C. Min, Semicond. Sci. Technol. 27, 083001 (2012).

${ }^{15}$ T. Fujii, M. Kawasaki, A. Sawa, Y. Kawazoe, H. Akoh, and Y. Tokura, Phys. Rev. B 75, 165101 (2007).

${ }^{16}$ T. Uhrmann, T. Dimopoulos, A. Kovacs, A. Kohn, S. Weyers, U. Paschen, J. Smoliner, and H. Brückl, J. Phys. D: Appl. Phys. 42, 145114 (2009).

${ }^{17}$ B.-C. Min, J. C. Lodder, R. Jansen, and K. Motohashi, J. Appl. Phys. 99, 08S701 (2006).

${ }^{18}$ S. Suzuki, T. Yamamoto, H. Suzuki, K. Kawaguchi, and K. Takahashi, J. Appl. Phys. 81, 6830 (1997).

${ }^{19}$ T. Susaki, Y. Kozuka, Y. Tateyama, and H. Y. Hwang, Phys. Rev. B 76, 155110 (2007).

${ }^{20}$ R. C. Neville, B. Hoeneisen, and C. A. Mead, J. Appl. Phys. 43, 2124 (1972).

${ }^{21}$ J. Shiogai, M. Ciorga, M. Utz, D. Schuh, M. Kohda, D. Bougeard, T. Nojima, J. Nitta, and D. Weiss, Phys. Rev. B 89, 081307(R) (2014).

${ }^{22}$ A. Dankert, R. S. Dulal, and S. P. Dash, Sci. Rep. 3, 3196 (2013).

${ }^{23}$ C. Şahin, G. Vignale, and M. E. Flatté, Phys. Rev. B 89, 155402 (2014).

${ }^{24}$ S. P. Dash, S. Sharma, J. C. Le Breton, J. Peiro, H. Jaffres, J.-M. George, A. Lemaître, and R. Jansen, Phys. Rev. B 84, 054410 (2011).

${ }^{25}$ S. Sharma, S. P. Dash, H. Saito, S. Yuasa, B. J. van Wees, and R. Jansen, Phys. Rev. B 86, 165308 (2012).

${ }^{26}$ H. Nakamura, T. Koga, and T. Kimura, Phys. Rev. Lett. 108, 206601 (2012).

${ }^{27}$ T. Uemura, K. Kondo, J. Fujisawa, K. Matsuda, and M. Yamamoto, Appl. Phys. Lett. 101, 132411 (2012).

${ }^{28}$ S. Sharma, A. Spiesser, S. P. Dash, S. Iba, S. Watanabe, B. J. van Wees, H. Saito, S. Yuasa, and R. Jansen, Phys. Rev. B 89, 075301 (2014).

${ }^{29}$ O. Txoperena, M. Gobbi, A. Bedoya-Pinto, F. Golmar, X. Sun, L. E. Hueso, and F. Casanova, Appl. Phys. Lett. 102, 192406 (2013). 